\title{
Corps engendré par les points de 13-torsion des courbes elliptiques
}

\author{
par \\ Marusia Rebolledo Hochart (Paris)
}

Soit $E$ une courbe elliptique sur un corps de nombres. Les propriétés des accouplements de Weil montrent que le corps $K_{n}(E)$ engendré par les points de $n$-torsion de $E$ est une extension du corps $\mathbb{Q}\left(\mu_{n}\right)$ engendré par les racines $n$-ièmes de l'unité dans une clôture algébrique $\overline{\mathbb{Q}}$ de $\mathbb{Q}$.

Soit $\mathcal{S}$ l'ensemble des nombres premiers $p$ pour lesquels il existe une courbe elliptique $E$ telle que $K_{p}(E)=\mathbb{Q}\left(\mu_{p}\right)$. Il est connu que l'ensemble $\mathcal{S}$ contient les nombres 2, 3, 5 et Halberstadt a prouvé que 7 n'est pas dans $\mathcal{S}$. Merel a étudié plus avant cet ensemble. En particulier, il a montré avec Stein ([7], [9]) qu'aucun nombre premier $p \neq 13,7<p<1000$, n'appartient à $\mathcal{S}$. L'objet de ce papier est de traiter le cas $p=13$, pour lequel les techniques de Merel ne s'appliquent pas.

Nous démontrons le théorème suivant :

ThÉORÈme 1. Aucune courbe elliptique sur un corps de nombres n'a tous ses points d'ordre 13 définis sur $\mathbb{Q}\left(\mu_{13}\right)$ (autrement dit, $13 \notin \mathcal{S}$ ).

Notons $Y(13)$ (resp. $Y_{1}(13)$ ) la courbe affine sur $\mathbb{Q}$ classifiant les classes d'isomorphismes de paires $(E, \pi)$ (resp. $(E, P))$ où $E$ est une courbe elliptique et $\pi:(\mathbb{Z} / 13 \mathbb{Z})^{2} \hookrightarrow E[13]$ un plongement (resp. $P$ un point d'ordre 13 de $E$ ). Soit $X(13)$ (resp. $\left.X_{1}(13)\right)$ la courbe complète obtenue en adjoignant les pointes à $Y(13)\left(\right.$ resp. $\left.Y_{1}(13)\right)$.

Montrer le théorème revient à montrer que $Y(13)$ n'a pas de point $\mathbb{Q}\left(\mu_{13}\right)$-rationnel. Pour ce faire, nous étudions la courbe $Y_{1}(13)$ : l'examen détaillé d'un plongement de $X_{1}(13)$ dans sa jacobienne $J_{1}(13)$ et la description complète du groupe $J_{1}(13)\left(\mathbb{Q}\left(\mu_{13}\right)\right)$ permettent de borner le cardinal de $Y_{1}(13)\left(\mathbb{Q}\left(\mu_{13}\right)\right)$. On raisonne alors par l'absurde : l'image d'un point de $Y(13)\left(\mathbb{Q}\left(\mu_{13}\right)\right)$ par le revêtement $X(13) \rightarrow X_{1}(13)$ fournirait "trop" de points de $Y_{1}(13)\left(\mathbb{Q}\left(\mu_{13}\right)\right)$.

2000 Mathematics Subject Classification: Primary 11G05, 11F67; Secondary 11G18, $11 \mathrm{G} 40$. 
Nous noterons par la suite $\bar{n}$ l'image dans $\mathbb{Z} / 13 \mathbb{Z}$ d'un entier $n$, et $\widetilde{a}$ un relevé dans $\mathbb{Z}$ d'un entier a modulo 13. Soient $\Gamma_{0}(13), \Gamma=\Gamma_{1}(13)$ les sous-groupes de $\mathrm{SL}_{2}(\mathbb{Z})$ formés des éléments congrus respectivement à $\left(\begin{array}{l}\star \\ 0 \\ \star\end{array}\right)$ et $\left(\begin{array}{ll}1 & \star \\ 0 & 1\end{array}\right)$ modulo 13 . Nous noterons $S=S_{2}\left(\Gamma_{1}(13)\right)$ l'espace des formes modulaires paraboliques de poids 2 pour $\Gamma_{1}(13)$. Pour $\phi:(\mathbb{Z} / 13 \mathbb{Z})^{\times} \rightarrow \mathbb{C}^{\times}$ un caractère, $S_{2}(13, \phi)$ désignera l'ensemble des formes $f$ de $S$ telles que, pour tout $\left(\begin{array}{ll}a & b \\ c & d\end{array}\right)$ dans $\Gamma_{0}(13), f \mid\left(\begin{array}{ll}a & b \\ c & d\end{array}\right)=\phi(\bar{d}) . f$. Nous noterons enfin $W_{13}$ l'opérateur d'Atkin-Lehner, c'est-à-dire l'involution de $X_{1}(13)$ déduite de l'involution $z \mapsto-\frac{1}{13 z}$ sur $\overline{\mathcal{H}}$, où $\overline{\mathcal{H}}=\mathcal{H} \cup \mathbb{P}^{1}(\mathbb{Q}), \mathcal{H}$ étant le demi-plan de Poincaré.

1. Étude du groupe $J_{1}(13)\left(\mathbb{Q}\left(\mu_{13}\right)\right)$. Rappelons que deux pointes

$$
\alpha_{i}=p_{i} / q_{i}, \quad i=1,2, \quad \operatorname{pgcd}\left(p_{i}, q_{i}\right)=1,
$$

sont équivalentes modulo $\Gamma=\Gamma_{1}(13)$ si et seulement si

$$
q_{2}=\lambda q_{1}(\bmod 13), \quad p_{2}=\lambda p_{1}\left(\bmod \operatorname{pgcd}\left(q_{1}, 13\right)\right), \quad \lambda= \pm 1
$$

(voir [2]). De plus les pointes de $X_{1}(13)$ sont toutes $\mathbb{Q}\left(\mu_{13}\right)$-rationnelles et $\sigma \in(\mathbb{Z} / 13 \mathbb{Z})^{\times} \cong \operatorname{Gal}\left(\mathbb{Q}\left(\mu_{13}\right) / \mathbb{Q}\right)$ opère sur une pointe par $\left(\begin{array}{ll}\sigma & 0 \\ 0 & 1\end{array}\right)$. Ainsi la courbe $X_{1}(13)$ possède six pointes $\mathbb{Q}$-rationnelles : $P_{i}=\Gamma \cdot \frac{13}{i}, 1 \leq i \leq 6$, et six autres pointes : $Q_{j}=\Gamma \cdot \frac{j}{13}, 1 \leq j \leq 6$. L'opérateur d'Atkin-Lehner $W_{13}$ échange $P_{i}$ et $Q_{i}$ pour $1 \leq i \leq 6$.

Choisissons le plongement de $X_{1}(13)$ dans $J_{1}(13)$ donné par la pointe $P_{6}$ :

$$
\iota: X_{1}(13) \rightarrow J_{1}(13), \quad P \mapsto\left[(P)-\left(P_{6}\right)\right] .
$$

Ogg $([10])$ montre que le sous-groupe de $J_{1}(13)\left(\mathbb{Q}\left(\mu_{13}\right)\right)$ engendré par les images sous $\iota$ des pointes $\mathbb{Q}$-rationnelles :

$$
C_{1}=\left\langle u_{1}, \ldots, u_{6}\right\rangle, \quad u_{i}=\iota\left(P_{i}\right), \quad 1 \leq i \leq 6,
$$

est cyclique d'ordre 19. Par conséquent, le sous-groupe

$$
C_{2}=W_{13} \cdot C_{1}=\left\langle v_{1}, \ldots, v_{6}\right\rangle, \quad v_{j}=\left[\left(Q_{j}\right)-\left(Q_{6}\right)\right], \quad 1 \leq j \leq 6,
$$

l'est également. Ces deux groupes d'ordre 19, distincts pour des raisons de rationalité, sont donc en somme directe et on a

$$
(\mathbb{Z} / 19 \mathbb{Z})^{2} \cong C_{1} \oplus C_{2} \subset J_{1}(13)\left(\mathbb{Q}\left(\mu_{13}\right)\right) .
$$

On va en fait montrer l'égalité :

Proposition 1. On a $J_{1}(13)\left(\mathbb{Q}\left(\mu_{13}\right)\right)=C_{1} \oplus C_{2}$.

L'essentiel de la démonstration repose sur le lemme suivant :

LeMme 1. Le groupe $J_{1}(13)\left(\mathbb{Q}\left(\mu_{13}\right)\right)$ est fini.

D'après ce qui précède, le lemme suivant suffira alors à prouver la proposition : 
Lemme 2. Pour tout premier $l$ distinct de 19, on a

$$
J_{1}(13)\left(\mathbb{Q}\left(\mu_{13}\right)\right)[l]=\{0\},
$$

et pour tout $n \in \mathbb{N}$,

$$
J_{1}(13)\left(\mathbb{Q}\left(\mu_{13}\right)\right)\left[19^{n}\right]=J_{1}(13)\left(\mathbb{Q}\left(\mu_{13}\right)\right)[19] \cong(\mathbb{Z} / 19 \mathbb{Z})^{2} .
$$

1.1. Finitude de $J_{1}(13)\left(\mathbb{Q}\left(\mu_{13}\right)\right)$. Rappelons que la courbe $X_{1}(13)$ est de genre $g=2$. Le groupe $\Gamma_{0}(13) / \Gamma_{1}(13) \cong(\mathbb{Z} / 13 \mathbb{Z})^{\times}$agissant sur $S$, on a $S=\bigoplus_{\phi} S_{2}(13, \phi)$, où $\phi$ décrit l'ensemble des caractères pairs de $(\mathbb{Z} / 13 \mathbb{Z})^{\times}$ (voir [3]).

La formule de Riemann-Hurwitz montre que $S_{2}(13, \phi)=0$ pour $\phi$ d'ordre distinct de l'ordre maximal 6. En effet, si $\Gamma_{1}(13) \subset \operatorname{ker} \phi \subset \Gamma_{0}(13)$ sont des inclusions strictes, la courbe modulaire associée à ker $\phi$ est de genre nul. Notons $\varepsilon, \bar{\varepsilon}$ les deux caractères d'ordre 6 de $(\mathbb{Z} / 13 \mathbb{Z})^{\times}$, et $\zeta$ une racine primitive douzième de l'unité. Ces deux caractères sont définis par $\varepsilon(2)=\zeta^{2}, \bar{\varepsilon}(2)=\zeta^{-2}$. On a $S=S_{2}(13, \varepsilon) \oplus S_{2}(13, \bar{\varepsilon})$, et les $\mathbb{C}$-espaces vectoriels $S_{2}(13, \varepsilon)$ et $S_{2}(13, \bar{\varepsilon})$ sont de dimension 1 engendrés chacun par une forme primitive $f_{\varepsilon}$ et $f_{\bar{\varepsilon}}$ respectivement.

Les résultats de Kato ([4, corollaire 14.3]) en direction de la conjecture de Birch et Swinnerton-Dyer montrent que si $L\left(J_{1}(13), \mathbb{Q}\left(\mu_{13}\right), 1\right) \neq 0$ alors $J_{1}(13)\left(\mathbb{Q}\left(\mu_{13}\right)\right)$ est fini. De plus, d'après un théorème de Shimura complété par Carayol,

$$
L\left(J_{1}(13), \mathbb{Q}\left(\mu_{13}\right), s\right)=\prod_{\chi, f} L(f, \chi, s),
$$

où $\chi$ décrit l'ensemble des caractères de Dirichlet modulo 13 et $f$ l'ensemble des formes primitives de poids 2 de niveau 13. D'après ce qui précède, on a donc

$$
L\left(J_{1}(13), \mathbb{Q}\left(\mu_{13}\right), 1\right)=\prod_{\chi:(\mathbb{Z} / 13 \mathbb{Z})^{\times} \rightarrow \mathbb{C}^{\times}} L\left(f_{\varepsilon}, \chi, 1\right) \cdot L\left(f_{\bar{\varepsilon}}, \chi, 1\right) .
$$

Pour prouver la finitude du groupe $J_{1}(13)\left(\mathbb{Q}\left(\mu_{13}\right)\right)$, il suffit alors de prouver que la condition $\left(\mathrm{C}_{1}\right)$ suivante est satisfaite :

$\left(\mathrm{C}_{1}\right) \quad \forall \chi:(\mathbb{Z} / 13 \mathbb{Z})^{\times} \rightarrow \mathbb{C}^{\times}, \quad L\left(f_{\varepsilon}, \chi, 1\right) \neq 0, \quad L\left(f_{\bar{\varepsilon}}, \chi, 1\right) \neq 0$.

Pour $\chi:(\mathbb{Z} / 13 \mathbb{Z})^{\times} \rightarrow \mathbb{C}^{\times}$, notons $\tau(\chi)=\sum_{b \bmod 13} \chi(b) e^{-2 i \pi \tilde{b} / 13}$ la somme de Gauss. La formule ([6, théorèmes 3.9 et 4.2$])$

$$
L(f, \chi, 1)=\frac{-2 i \pi \tau(\chi)}{13} \sum_{a \bmod 13} \bar{\chi}(a) \int_{\widetilde{a} / 13}^{\infty} f(u) d u \quad(f \in S)
$$

nous conduit à utiliser les symboles modulaires. Nous allons énoncer une condition $\left(\mathrm{C}_{2}\right)$ sur certains symboles modulaires, qui entraîne $\left(\mathrm{C}_{1}\right)$ et donc la finitude de $J_{1}(13)\left(\mathbb{Q}\left(\mu_{13}\right)\right)$. 
1.1.1. Symboles modulaires et condition suffisante à la finitude de $J_{1}(13)\left(\mathbb{Q}\left(\mu_{13}\right)\right)$. Dans cette section, nous verrons $X=X_{1}(13)(\mathbb{C})$ comme la surface de Riemann compacte connexe $\Gamma \backslash \overline{\mathcal{H}}$, où $\overline{\mathcal{H}}=\mathcal{H} \cup \mathbb{P}^{1}(\mathbb{Q}), \mathcal{H}$ étant le demi-plan de Poincaré. Pour ce qui concerne la théorie des symboles modulaires nous renvoyons à $[2],[6],[8]$.

Soient $H_{1}(X ; \mathbb{Z})\left(\right.$ resp. $H_{1}(X$, ptes; $\left.\mathbb{Z})\right)$ l'homologie singulière absolue (resp. relative à l'ensemble $\{$ ptes $\}$ des pointes) de $X$. Pour $\alpha, \beta \in \mathbb{P}^{1}(\mathbb{Q})$, on note $\{\alpha, \beta\}$, appelé symbole modulaire, la classe d'homologie dans $H_{1}(X$, ptes; $\mathbb{Z})$ de l'image d'une géodésique reliant $\alpha$ à $\beta$.

Notons $H=H_{1}(X ; \mathbb{C})$ et $H^{\prime}=H_{1}(X$, ptes; $\mathbb{C})$. Le $\mathbb{C}$-espace vectoriel $H$ est de dimension $2 g=4$, et vérifie la suite exacte longue d'homologie :

$$
0 \rightarrow H \rightarrow H^{\prime} \stackrel{\delta}{\rightarrow} \mathbb{C}[\text { ptes }] \stackrel{\text { deg }}{\rightarrow} \mathbb{C} \rightarrow 0,
$$

où $\delta$ est l'application "bord" : $\{\alpha, \beta\} \mapsto(\Gamma \beta)-(\Gamma \alpha)$, et deg l'application "degré" usuelle sur les diviseurs. L'espace vectoriel $H^{\prime}$ est donc de dimension 15 sur $\mathbb{C}$.

On dispose également de l'homomorphisme de groupes de Manin :

$$
\xi:\left\{\begin{array}{l}
\mathbb{C}\left[\Gamma \backslash \mathrm{SL}_{2}(\mathbb{Z})\right] \rightarrow H^{\prime}, \\
{[\Gamma . g] \mapsto\{g 0, g \infty\}=\{b / d, a / c\}, \quad \text { avec } \quad g=\left(\begin{array}{ll}
a & b \\
c & d
\end{array}\right) \in \mathrm{SL}_{2}(\mathbb{Z}) .}
\end{array}\right.
$$

Rappelons que $\xi$ est surjectif, de noyau engendré par les relations de Manin, c'est-à-dire par les éléments de la forme $[x]+[x \sigma],[x]+[x \tau]+\left[x \tau^{2}\right]$, où $x \in \Gamma \backslash \mathrm{SL}_{2}(\mathbb{Z})$, et $\sigma, \tau$ des éléments de $\mathrm{SL}_{2}(\mathbb{Z})$ d'ordre respectif 4 et 3 :

$$
\sigma=\left(\begin{array}{rr}
0 & -1 \\
1 & 0
\end{array}\right), \quad \tau=\left(\begin{array}{ll}
0 & -1 \\
1 & -1
\end{array}\right)
$$

Notons $\mathcal{A}=\left[(\mathbb{Z} / 13 \mathbb{Z})^{2}-(0,0)\right]$. Remarquons que l'application

$$
\Gamma \cdot\left(\begin{array}{ll}
a & b \\
c & d
\end{array}\right) \mapsto(\bar{c}, \bar{d})
$$

est une bijection de $\Gamma \backslash \mathrm{SL}_{2}(\mathbb{Z})$ sur $\mathcal{A}$. Le morphisme de Manin fournit donc une application encore notée $\xi$ sur $\mathbb{C}[\mathcal{A}]$. On note $[c, d]$, appelé symbole de Manin, l'image par $\xi$ dans $H^{\prime}$ de l'élément $(c, d)$ de $\mathcal{A}$. Le groupe $\mathrm{SL}_{2}(\mathbb{Z})$ agit sur les symboles de Manin par multiplication à droite.

Les correspondances de Hecke $T_{n}, n \in \mathbb{N}$, et les opérateurs diamants $\langle m\rangle,(m, 13)=1$, sur $X_{1}(13)$, induisent des endomorphismes de $H^{\prime}$ que nous noterons respectivement $T_{n}^{\prime}$ et $\langle m\rangle^{\prime}$ : on pose $t^{\prime} .\{\alpha, \beta\}=\{t . \alpha, t . \beta\}$, où $\alpha, \beta \in \mathbb{P}^{1}(\mathbb{Q}), t=T_{n}$ ou $\langle m\rangle$. Soit $\mathbb{T}^{\prime}$ la sous-algèbre de $\operatorname{End}\left(H^{\prime}\right)$ engendrée par les endomorphismes $T_{p}^{\prime}$, et $\langle q\rangle^{\prime}$ pour $p, q$ premiers, $q \neq 13$. L'espace $H$ vu comme sous-espace de $H^{\prime}$ est stable sous l'action de $\mathbb{T}^{\prime}$. L'action des opérateurs diamants est donnée par

$$
\langle m\rangle^{\prime}[c, d]=[\bar{m} c, \bar{m} d] .
$$


Pour $\chi$ caractère de Dirichlet modulo 13 et $x$ dans $H^{\prime}$, notons $H^{\prime} \chi$ (resp. $H^{\chi}$ ) la composante $\chi$-isotypique de $H^{\prime}$ (resp. $H$ ), et $x^{\chi}$ la projection de $x$ sur $H^{\prime \chi}$. Posons également

$\theta_{\chi}=t_{\chi} \cdot \sum_{a \bmod 13} \chi(a) \cdot[1, a] \quad$ avec $\quad t_{\chi}= \begin{cases}1 & \text { si } \chi \text { est impair } \\ \left(T_{2}^{\prime}-2\langle 2\rangle^{\prime}-1\right) & \text { si } \chi \text { est pair. }\end{cases}$

LEMme 3. La condition $\left(\mathrm{C}_{2}\right)$ suivante entraîne la condition $\left(\mathrm{C}_{1}\right)$ et donc la finitude du groupe $J_{1}(13)\left(\mathbb{Q}\left(\mu_{13}\right)\right)$ :

$$
\forall \chi:(\mathbb{Z} / 13 \mathbb{Z})^{\times} \rightarrow \mathbb{C}^{\times}, \quad \theta_{\chi}^{\varepsilon} \neq 0, \quad \theta_{\chi}^{\bar{\varepsilon}} \neq 0 .
$$

Preuve. Montrons tout d'abord que l'élément $\theta_{\chi}$ de $H^{\prime}$ est en réalité dans $H$. On a

$$
\delta\left(\theta_{\chi}\right)=\delta\left(t_{\chi} \cdot \sum_{a \bmod 13} \chi(a) \cdot\left\{\frac{-1}{\widetilde{a}}, 0\right\}\right)=-\sum_{a \bmod 13} \chi(a)\left(t_{\chi}\left(\Gamma \cdot \frac{-1}{\widetilde{a}}\right)\right) .
$$

Or

$$
\Gamma \cdot\left(\frac{-1}{\widetilde{a}}\right)=\Gamma \cdot \frac{1}{\widetilde{a}}=\Gamma \cdot\left(\frac{-1}{-\widetilde{a}}\right),
$$

donc pour $\chi$ impair,

$$
\delta\left(\theta_{\chi}\right)=-\sum_{k=1}^{6}(\chi(\bar{k})+\chi(-\bar{k}))\left(\Gamma \cdot \frac{1}{k}\right)=0 .
$$

Pour $\chi$ pair, un calcul montre que $t_{\chi}\left(\Gamma \cdot \frac{1}{\tilde{a}}\right)=0([11,2.4])$.

La conjugaison complexe sur $X$, déduite de l'involution $z \mapsto-\bar{z}$ sur $\mathcal{H}$, induit sur $H^{\prime}$ l'involution $i:[c, d] \mapsto[-c, d]$. Le sous-espace $H$ est stable sous $i$. Notons $H^{+}$(resp. $H^{-}$) la partie invariante (resp. antiinvariante) de $H$ sous $i$. On a $H=H^{+} \oplus H^{-}$, et $H^{+}, H^{-}$sont des $\mathbb{C}$-espaces vectoriels de dimension 2. On vérifie que $\theta_{\chi} \in H^{+}$pour $\chi$ pair, et $\theta_{\chi} \in H^{-}$pour $\chi$ impair.

On dispose d'autre part de l'application $\mathbb{C}$-linéaire

$$
H \rightarrow \operatorname{Hom}_{\mathbb{C}}\left(H^{0}\left(X, \Omega_{X}^{1}\right), \mathbb{C}\right), \quad c \mapsto\left(\omega \mapsto \int_{c} \omega\right) .
$$

Pour $f \in S=S_{2}\left(\Gamma_{1}(13)\right)$, notons $\omega_{f}$ la différentielle holomorphe sur $X_{1}(13)$ induite par $2 i \pi f(z) d z$. L'application $f \mapsto \omega_{f}$ est un isomorphisme de $S$ sur $H^{0}\left(X, \Omega_{X}^{1}\right)$. Le morphisme qui s'en déduit,

$$
F: H \rightarrow \operatorname{Hom}_{\mathbb{C}}(S, \mathbb{C}), \quad c \mapsto\left(f \mapsto \int_{c} \omega_{f}\right),
$$

induit un isomorphisme $F^{+}$sur $H^{+}$, resp. $F^{-}$sur $H^{-}$.

Soit $\mathbb{T}$ l'algèbre engendrée par les correspondances de Hecke $T_{p}$ et les diamants $\langle q\rangle$ sur $X_{1}(13)$, pour $p, q$ premiers, $q \neq 13$. L'algèbre $\mathbb{T}$ agit à droite sur les formes modulaires et donc $\operatorname{sur} \operatorname{Hom}_{\mathbb{C}}(S, \mathbb{C})$. Cette action munit $H^{+}$, 
resp. $H^{-}$, d'une structure de $\mathbb{T}$-module : pour $t \in \mathbb{T}, c \in H^{ \pm}$, t.c est défini par $\int_{t . c} \omega_{f}=\int_{c} \omega_{t . f}$. Les actions de $\mathbb{T}$ et $\mathbb{T}^{\prime}$ sur $H$ coïncident. En particulier l'action des diamants fournit des isomorphismes sur chaque composante : $F^{ \pm, \phi}: H^{ \pm, \phi} \stackrel{\sim}{\rightarrow} \operatorname{Hom}_{\mathbb{C}}\left(S_{2}(13, \phi), \mathbb{C}\right)$, pour $\phi$ caractère modulo 13 . On en déduit $H^{\phi}=0$ pour $\phi \neq \varepsilon, \bar{\varepsilon}$, et $H=H^{\varepsilon} \oplus H^{\bar{\varepsilon}}$.

Soient

$$
c_{\chi}=\sum_{a \bmod 13} \bar{\chi}(a)\left\{\frac{\tilde{a}}{13}, \infty\right\} \in H^{\prime}, \quad f \in S, \quad \chi:(\mathbb{Z} / 13 \mathbb{Z})^{\times} \rightarrow \mathbb{C}^{\times} .
$$

On a

$$
L(f, \chi, 1)=-\frac{\tau(\chi)}{13} \int_{c_{\chi}} \omega_{f}
$$

On remarque que $\theta_{\chi}=t_{\chi} W_{13} c_{\bar{\chi}}$. Notons $t_{\chi}^{*}$ l'élément de $\mathbb{T}$ tel que l'on a $t_{\chi} W_{13}=W_{13} t_{\chi}^{*}$.

Supposons à présent que la condition $\left(\mathrm{C}_{2}\right)$ est vérifiée. En particulier $\theta_{\chi}^{\varepsilon} \neq 0$. Les isomorphismes $F^{ \pm, \varepsilon}$ montrent qu'il existe $f$ dans $S_{2}(13, \varepsilon)$ telle que $\int_{\theta_{\chi}^{\varepsilon}} \omega_{f} \neq 0$. L'espace $S_{2}(13, \varepsilon)$ étant engendré par $f_{\varepsilon}$, il s'ensuit que $\int_{\theta_{\chi}} \omega_{f_{\varepsilon}}=\int_{\theta_{\chi}^{\varepsilon}} \omega_{f_{\varepsilon}} \neq 0$. Or

$$
\int_{\theta_{\chi}} \omega_{f_{\varepsilon}}=\int_{t_{\chi} \cdot W_{13} c_{\bar{\chi}}} \omega_{f_{\varepsilon}}=\int_{t_{\chi}^{*} \cdot c_{\bar{\chi}}} \omega_{f_{\bar{\varepsilon}}}=\lambda_{\chi} \cdot \int_{c_{\bar{\chi}}} \omega_{f_{\bar{\varepsilon}}}=-\frac{13 \lambda_{\chi}}{\tau(\bar{\chi})} L\left(f_{\bar{\varepsilon}}, \bar{\chi}, 1\right),
$$

où $t_{\chi}^{*} \cdot f_{\bar{\varepsilon}}=\lambda_{\chi} \cdot f_{\bar{\varepsilon}}, \lambda_{\chi} \in \mathbb{C}$. Ceci prouve que si $\theta_{\chi}^{\varepsilon} \neq 0$ alors $L\left(f_{\bar{\varepsilon}}, \bar{\chi}, 1\right) \neq 0$. De même, si $\theta_{\chi}^{\bar{\varepsilon}} \neq 0$ alors $L\left(f_{\varepsilon}, \bar{\chi}, 1\right) \neq 0$.

1.1.2. Vérification de la condition $\left(\mathrm{C}_{2}\right)$. Nous allons faire les calculs dans les composantes $H^{\prime \varepsilon}, H^{\prime \bar{\varepsilon}}$ de $H^{\prime}$, espace pour lequel nous disposons de la présentation de Manin. Pour cela nous allons déterminer une base de $H^{\prime \varepsilon}$ (resp. $H^{\prime \bar{\varepsilon}}$ ) et exprimer $\theta_{\chi}^{\varepsilon}$ (resp. $\theta_{\chi}^{\bar{\varepsilon}}$ ) dans cette base. La dimension de ces composantes se déduit de la suite exacte $(*)$ de la section 1.1.1 :

Lemme 4. On a $\operatorname{dim} H^{\prime}=15$, et $\operatorname{dim} H^{\prime \phi}$ vaut 0 si $\phi$ est impair, 1 si $\phi$ est trivial, 4 si $\phi=\varepsilon$ ou $\bar{\varepsilon}$, et 2 sinon.

Soit $\phi$ désignant indifféremment $\varepsilon$ ou $\bar{\varepsilon}$. On peut voir $H^{\prime \phi}$ comme le quotient de $H^{\prime}$ par les relations

$$
[n u, n v]=\phi(n) \cdot[u, v] \quad\left(n \in(\mathbb{Z} / 13 \mathbb{Z})^{\times},(u, v) \in \mathcal{A}\right) .
$$

Notons $[u, v]^{\phi}$ l'image de $[u, v]$ dans $H^{\prime \phi}$. Pour tout $u \neq 0$ dans $\mathbb{Z} / 13 \mathbb{Z}$, on a $[u, v]^{\phi}=\phi(u) \cdot\left[1, v u^{-1}\right]^{\phi}$, et $[0, v]^{\phi}=\phi(v) \cdot[0,1]^{\phi}$. On en déduit que $\left\{[0,1]^{\phi},[1, w]^{\phi}: w \in \mathbb{Z} / 13 \mathbb{Z}\right\}$ forme un système de générateurs de $H^{\prime \phi}$. En écrivant les relations de Manin pour ces générateurs, on obtient la proposition suivante : 
Lemme 5. Les éléments $[1,0]^{\phi},[1,2]^{\phi},[1,3]^{\phi},[1,-3]^{\phi}$ forment une base de $H^{\prime \phi}$, où $\phi=\varepsilon$ ou $\bar{\varepsilon}$. Dans cette base, les générateurs $[0,1]^{\phi},[1, w]^{\phi}$, pour $w \in \mathbb{Z} / 13 \mathbb{Z}$, s'écrivent respectivement :

$$
\begin{array}{ll}
{[0,1]^{\phi}=-[1,0]^{\phi},} & {[1,1]^{\phi}=[1,-1]^{\phi}=0,} \\
{[1,-2]^{\phi}=[1,2]^{\phi},} & {[1,6]^{\phi}=[1,-6]^{\phi}=-\phi(6) \cdot[1,2]^{\phi},} \\
{[1,4]^{\phi}=-\phi(4) \cdot[1,3]^{\phi},} & {[1,-4]^{\phi}=-\phi(4) \cdot[1,-3]^{\phi},} \\
{[1,5]^{\phi}=\phi(6) \cdot[1,3]^{\phi}-\phi(6) \cdot[1,2]^{\phi},} & {[1,-5]^{\phi}=\phi(4) \cdot[1,2]^{\phi}-\phi(4) \cdot[1,-3]^{\phi} .}
\end{array}
$$

Pour $\chi$ impair, on a alors :

$$
\begin{aligned}
\theta_{\chi}^{\phi}= & (\chi(3)-\chi(4) \phi(4)+\chi(5) \phi(6)) \cdot[1,3]^{\phi} \\
& +(-\chi(3)+\chi(4) \phi(4)+\chi(5) \phi(4)) \cdot[1,-3]^{\phi} .
\end{aligned}
$$

Or, $\chi$ est défini par $\chi(2)=\zeta^{k}, \zeta$ une racine primitive douzième de l'unité, $k=1,3,5,7,9,11$. Donc $\chi(3)-\chi(4) \varepsilon(4)+\chi(5) \varepsilon(6)=\zeta^{4 k}-\zeta^{2 k+4}+\zeta^{9 k+10}$. Ce terme est non nul pour $k=1,3,5,7,9,11$, donc $\theta_{\chi}^{\varepsilon}$ est non nul pour $\chi$ impair. De même, on montre que $\theta_{\chi}^{\bar{\varepsilon}} \neq 0$ pour $\chi$ impair.

D'après $[8]$, on a $T_{2}^{\prime} \cdot[c, d]=[2 c, d]+[2 c, c+d]+[c+d, 2 d]+[c, 2 d]$. Donc, pour $\chi$ pair,

où

$$
\begin{aligned}
\theta_{\chi}^{\phi}= & \sum_{a=1}^{12} \chi(a) \cdot\left([2, a]^{\phi}+[2,1+a]^{\phi}+[1+a, 2 a]^{\phi}+[1,2 a]^{\phi}\right. \\
& \left.-2[2,2 a]^{\phi}-[1, a]^{\phi}\right), \\
\theta_{\chi}^{\phi}= & A_{\phi} \cdot[1,2]^{\phi}+B_{\phi} \cdot[1,3]^{\phi}+C_{\phi} \cdot[1,-3]^{\phi},
\end{aligned}
$$

$$
\begin{aligned}
C_{\phi}= & {[\chi(2)(1-\phi(4)-\phi(2))-\chi(3) \phi(2)+\chi(4) \phi(5)} \\
& +\chi(5)(\phi(2)+\phi(4))+2 \chi(6) \phi(2)] .
\end{aligned}
$$

Si $\chi$ est défini par $\chi(2)=\zeta^{k}$, on a

$$
C_{\varepsilon}=\zeta^{k}\left(1-\zeta^{4}-\zeta^{2}\right)-\zeta^{4 k+2}-\zeta^{2 k+3}+\zeta^{9 k}\left(\zeta^{4}+\zeta^{2}\right)+2 \zeta^{5 k+2}
$$

Ce terme étant non nul pour $k=2,4,6,8,12, \theta_{\chi}^{\varepsilon} \neq 0$ pour $\chi$ pair. De même $C_{\bar{\varepsilon}} \neq 0$ montre que $\theta_{\chi}^{\bar{\varepsilon}} \neq 0$ pour $\chi$ pair.

Ceci termine la preuve de la finitude de $J_{1}(13)\left(\mathbb{Q}\left(\mu_{13}\right)\right)$ (c'est-à-dire du lemme 1).

1.2. Fin de la preuve de la proposition 1. Comme nous l'avons signalé au début de cette section, le groupe $J_{1}(13)\left(\mathbb{Q}\left(\mu_{13}\right)\right)$ étant fini et contenant $C_{1} \oplus C_{2}$ (voir 1.1), il suffit maintenant de montrer le lemme 2 pour achever la preuve de la proposition 1.

Preuve du lemme 2. Soient $p$ un nombre premier distinct de 13 et $\mathfrak{p}$ un idéal de $\mathbb{Z}\left[\mu_{13}\right]$ au-dessus de $p$. Notons $k_{p}$ (resp. $f_{p}=\left[k_{p}: \mathbb{F}_{p}\right]$ ) le corps (resp. le degré) résiduel en $\mathfrak{p}$ de $\mathbb{Z}\left[\mu_{13}\right], \bar{k}_{p}$ une clôture algébrique de $k_{p}$, et 
$\phi_{p} \in \operatorname{Gal}\left(\overline{\mathbb{F}}_{p} / \mathbb{F}_{p}\right)$ un endomorphisme de Frobenius en $p$. Le modèle de Néron $\mathcal{J}_{1}(13)$ sur $\mathbb{Z}$ de $J_{1}(13)$ a bonne réduction modulo $p$. Par conséquent, pour tout nombre premier $l$ distinct de $p$, on a

$$
J_{1}(13)\left(\mathbb{Q}\left(\mu_{13}\right)\right)[l] \hookrightarrow J_{1}(13)\left(k_{p}\right)[l] .
$$

Or $J_{1}(13)\left(k_{p}\right)[l]$ est l'ensemble des invariants sous $\phi_{p}^{f_{p}}$ de $J_{1}(13)\left(\bar{k}_{p}\right)[l]$ muni de sa structure de module galoisien. Montrons que pour $l \neq 19$ cet ensemble se réduit à $\{0\}$.

Pour $l \neq 2$, le $\mathbb{T} / l \mathbb{T}$-module $J_{1}(13)\left(\bar{k}_{p}\right)[l]$ est libre de rang 2 (se reporter à [13, théorème 3.4, corollaire 2]), et la relation d'Eichler-Shimura dans $\operatorname{End}_{\mathbb{T}}\left(J_{1}(13)\left(\bar{k}_{p}\right)[l]\right)$ (voir par exemple [12, théorème 2$]$ )

$$
\phi_{p}^{2}-T_{p} \phi_{p}+p\langle p\rangle=0
$$

permet de trouver un polynôme $P_{p} \in \mathbb{T} / l \mathbb{T}[X]$ annulant $\phi_{p}^{f_{p}}$. Si le $\mathbb{T} / l \mathbb{T}$ module $J_{1}(13)\left(\bar{k}_{p}\right)[l]$ admettait des invariants sous $\phi_{p}^{f_{p}}$ alors 1 serait racine de $P_{p}$. Un choix judicieux de $p$ permet de conclure. Nous utiliserons également le fait que $\mathbb{T} \cong \mathbb{Z}[Y] / \Phi_{6}(Y)$ en niveau $13([5])$.

Choisissons d'abord $p=3$. Soit $l$ premier, $l \neq 2,3$. On a $f_{3}=3$, et un calcul montre que le polynôme suivant annule $\phi_{3}^{3}$ :

$$
P_{3}=X^{2}+\left(9\langle 3\rangle T_{3}-T_{3}^{3}\right) X+27\langle 3\rangle^{3} .
$$

Dans $\mathbb{T} / l \mathbb{T} \cong \mathbb{F}_{l}[Y] / \bar{\Phi}_{6}(Y), T_{3}$ s'écrit $2 Y-2$ et $\langle 3\rangle=Y([5])$, donc

$$
P_{3}(1)=1+9 Y(2 Y-2)+27 Y^{3}=2 \times 19 .
$$

On en déduit que, pour $l \neq 2,3,19$, on a $J_{1}(13)\left(\mathbb{Q}\left(\mu_{13}\right)\right)[l]=\{0\}$.

Soit maintenant $p=5$ et $l \neq 2,5$. On a $f_{5}=4$ et $\phi_{5}^{4}$ est annulé par

$$
P_{5}=X^{2}+\left(20\langle 5\rangle T_{5}^{2}-T_{5}^{4}-50\langle 5\rangle^{2}\right) X+625\langle 5\rangle^{4} .
$$

Dans $\mathbb{T} / l \mathbb{T} \cong \mathbb{F}_{l}[Y] / \bar{\Phi}_{6}(Y), T_{5}=-2 Y+1$ et $\langle 5\rangle=-1$, donc

$$
P_{5}(1)=627=3 \times 11 \times 19,
$$

et pour $l \neq 2,3,5,11,19$, on a $J_{1}(13)\left(\mathbb{Q}\left(\mu_{13}\right)\right)[l]=\{0\}$.

Les choix de $p$ qui précèdent montrent que, pour $l \neq 2,3,19$, on a $J_{1}(13)\left(\mathbb{Q}\left(\mu_{13}\right)\right)[l]=\{0\}$. Pour le cas $l=3$, choisissons $p=79$. On a $f_{79}=1$ car $79 \equiv 1(\bmod 13)$. Le polynôme $P_{79}=X^{2}-T_{79} X+79\langle 79\rangle$ annule $\phi_{79}$. Dans $\mathbb{T} / 3 \mathbb{T} \cong \mathbb{F}_{3}[Y] / \bar{\Phi}_{6}(Y)$, on a $T_{79}=4$, $\langle 79\rangle=1$, donc $P_{79}(1)=76$. Or $76 \not \equiv 0(\bmod 3)$, donc $J_{1}(13)\left(\mathbb{Q}\left(\mu_{13}\right)\right)[3]=\{0\}$.

Examinons le cas de la 2-torsion. Considérons $\phi_{5}^{4}$ sur le $\mathbb{F}_{2}$-espace vectoriel $J_{1}(13)\left(\overline{\mathbb{F}}_{5}\right)[2]$ de dimension 4 . Le polynôme

$$
P_{5}=X^{2}+X+1 \in \mathbb{F}_{2}[X] \subset \mathbb{T} / 2 \mathbb{T}[X] \cong \mathbb{F}_{4}[X]
$$

annule $\phi_{5}^{4}$ et n'admet pas 1 pour racine. Donc $J_{1}(13)\left(\mathbb{Q}\left(\mu_{13}\right)\right)[2]=\{0\}$. 
Terminons par le cas de la 19-torsion. On a

$$
(\mathbb{Z} / 19 \mathbb{Z})^{2} \cong C_{1} \oplus C_{2} \subset J_{1}(13)\left(\mathbb{Q}\left(\mu_{13}\right)\right)[19] .
$$

D'autre part, le calcul de la borne de Weil pour $J_{1}(13)\left(\mathbb{F}_{3^{3}}\right)$ donne

$$
\left|J_{1}(13)\left(\mathbb{F}_{3^{3}}\right)\right| \leq 1587 \text {. }
$$

Or $19^{3}>1587$, donc $\left|J_{1}(13)\left(\mathbb{F}_{3^{3}}\right)\left[19^{\infty}\right]\right|=19^{k}$ avec $k \leq 2$. On en déduit que $\left|J_{1}(13)\left(\mathbb{Q}\left(\mu_{13}\right)\right)[19]\right|=\left|J_{1}(13)\left(\mathbb{Q}\left(\mu_{13}\right)\right)\left[19^{\infty}\right]\right|=19^{2}$.

\section{Borne pour le cardinal de $Y_{1}(13)\left(\mathbb{Q}\left(\mu_{13}\right)\right)$}

2.1. Revêtement de degré 2 de $X_{1}(13)$ et conséquences

Lemme 6. Soit $(i, j) \in\{1, \ldots, 6\}^{2}$. Aucune fonction rationnelle sur $X_{1}(13)$ n'a pour diviseur des pôles $P_{i}+Q_{j}$.

Preuve. Considérons la courbe modulaire $X_{2}(13)$ associée au sous-groupe d'indice 3 de $\Gamma_{0}(13)$ suivant :

$$
\Gamma_{2}(13)=\left\{\left(\begin{array}{ll}
a & b \\
c & d
\end{array}\right) \in \Gamma_{0}(13): a^{2} \equiv \pm 1(\bmod 13)\right\} .
$$

Cette courbe est de genre nul et induit les revêtements

$$
X_{1}(13) \stackrel{2}{\rightarrow} X_{2}(13) \stackrel{3}{\rightarrow} X_{0}(13) .
$$

Notons $f: X_{1}(13) \rightarrow X_{2}(13)$, et $\sigma_{5}$ l'élément de $\Gamma_{0}(13)$ congru à $\left(\begin{array}{ll}5^{-1} & 0 \\ 0 & \frac{5}{5}\end{array}\right)$ modulo 13 . Comme $\overline{5}^{2} \equiv-1(\bmod 13)$, on a $\sigma_{5} \in \Gamma_{2}(13)$. D'autre part, $\sigma_{5}$ agit sur les pointes de $X_{1}(13)$ par

$$
\sigma_{5} \cdot P_{i}=\Gamma \cdot \frac{13}{5 i}, \quad \sigma_{5} \cdot Q_{j}=\Gamma \cdot \frac{5 j}{13} .
$$

Donc $f\left(P_{1}\right)=f\left(P_{5}\right), f\left(P_{2}\right)=f\left(P_{3}\right), f\left(P_{4}\right)=f\left(P_{6}\right), f\left(Q_{1}\right)=f\left(Q_{5}\right), f\left(Q_{2}\right)$ $=f\left(Q_{3}\right), f\left(Q_{4}\right)=f\left(Q_{6}\right)$ sont des pointes de $X_{2}(13)$.

Soit $\psi$ un isomorphisme de $X_{2}(13)$ sur $\mathbb{P}^{1}$ qui envoie la pointe $f\left(P_{1}\right)$ sur $\infty \in \mathbb{P}^{1}$. La fonction $\widetilde{f}=\psi \circ f$ est un revêtement de degré 2 de $X_{1}(13)$ sur $\mathbb{P}^{1}$. Ce qui précède montre que la fonction rationnelle sur $X_{1}(13)$ induite par $\tilde{f}$ admet pour diviseur des pôles

$$
P_{1}+P_{5} \sim P_{2}+P_{3} \sim P_{4}+P_{6} \sim Q_{1}+Q_{5} \sim Q_{2}+Q_{3} \sim Q_{4}+Q_{6} .
$$

Une fonction rationnelle de diviseur des pôles $P_{i}+Q_{j},(i, j) \in\{1, \ldots, 6\}^{2}$, induirait un revêtement de degré 2 de $X_{1}(13)$ sur $\mathbb{P}^{1}$ distinct de $\widetilde{f}$, ses fibres au-dessus de $\infty$ étant distinctes. L'unicité d'un tel revêtement pour les courbes de genre 2 interdit cette éventualité.

2.2. Borne

Proposition 2. Le cardinal de $Y_{1}(13)\left(\mathbb{Q}\left(\mu_{13}\right)\right)$ est inférieur ou égal $\grave{a} 12^{2}$. 
Preuve. Rappelons que $J_{1}(13)\left(\mathbb{Q}\left(\mu_{13}\right)\right)=C_{1} \oplus C_{2}$, avec $C_{1}=\left\langle u_{1}, \ldots, u_{6}\right\rangle$ $\subset J_{1}(13)(\mathbb{Q}), C_{2}=W_{13} \cdot C_{1}=\left\langle v_{1}, \ldots, v_{6}\right\rangle, u_{i}=\iota\left(P_{i}\right), v_{j}=\left[\left(Q_{j}\right)-\left(Q_{6}\right)\right]$ pour $1 \leq i, j \leq 6$ (proposition 1 ). Les résultats de Ogg ([10]) montrent que $C_{1}, C_{2}$ sont des groupes cycliques d'ordre 19 , et on a $u_{i}=a_{i} u_{4}, v_{i}=a_{i} v_{4}$, $a_{i} \in \mathbb{Z} / 19 \mathbb{Z}$, avec $a_{1}=4, a_{2}=-5, a_{3}=6, a_{4}=1, a_{5}=-3, a_{6}=0$, où on note encore $n$ la classe d'un entier $n$ dans $\mathbb{Z} / 19 \mathbb{Z}$.

En particulier, il existe $(a, b) \in(\mathbb{Z} / 19 \mathbb{Z})^{2}$ tels que

$$
\iota\left(Q_{6}\right)=\left[\left(Q_{6}\right)-\left(P_{6}\right)\right]=a u_{4}+b v_{4} .
$$

Or $W_{13} . \iota\left(Q_{6}\right)=-\iota\left(Q_{6}\right)=b u_{4}+a v_{4}$, donc $b=-a$ dans $\mathbb{Z} / 19 \mathbb{Z}$. De plus, d'après 2.1 , on a $P_{4}+P_{6} \sim Q_{4}+Q_{6}$, donc $2 \iota\left(Q_{6}\right)=u_{4}-v_{4}=2 a u_{4}-2 a v_{4}$. On en déduit que $a=-9$ et $\iota\left(Q_{6}\right)=-9 u_{4}+9 v_{4}$.

Soit maintenant $P$ dans $Y_{1}(13)\left(\mathbb{Q}\left(\mu_{13}\right)\right)$. Notons $u=\iota(P)$ et soient $(\mu, \nu) \in(\mathbb{Z} / 19 \mathbb{Z})^{2}$ tels que $u=\mu u_{4}+\nu v_{4}$. Ogg montre que $C_{1} \cap \iota\left(Y_{1}(13)\right)=\emptyset$. On en déduit que $\nu \neq 0$. D'autre part, si $\mu=-9$, on a $u=\iota\left(Q_{6}\right)+(\nu-9) v_{4}$, donc $\left[(P)-\left(Q_{6}\right)\right] \in C_{2}$, c'est-à-dire $\iota\left(W_{13} . P\right) \in C_{1} \cap \iota\left(Y_{1}(13)\right)$. Ceci étant impossible, $\mu \neq-9$.

Supposons qu'il existe $i, j, k$ dans $\{1, \ldots, 6\}$ tels que $u=u_{i}+v_{j}-v_{k}$. On aurait alors $P+Q_{k} \sim P_{i}+Q_{j}$, ce qui est impossible d'après 2.1. La différence $a_{j}-a_{k}$ décrivant $\mathbb{Z} / 19 \mathbb{Z}$ lorsque $j, k$ décrivent $\{1, \ldots, 6\}$, ceci impose $\mu \neq 0,1,4,6,-3,-5$. De même, on montre que $u \neq u_{i}-u_{k}+v_{j}+\iota\left(Q_{6}\right)$, ce qui impose $\nu \neq 9,-9,6,-6,4,-4$.

Les contraintes précédentes sur $(\mu, \nu)$ montrent la proposition.

3. Preuve du théorème. Il s'agit de montrer que $Y_{1}(13)\left(\mathbb{Q}\left(\mu_{13}\right)\right)$ est vide. Procédons par l'absurde : soit $(E, \pi)$ un point de $Y(13)\left(\mathbb{Q}\left(\mu_{13}\right)\right)$, où $E$ est une courbe elliptique définie sur $\mathbb{Q}\left(\mu_{13}\right)$ et $\pi$ est un plongement $(\mathbb{Z} / 13 \mathbb{Z})^{2} \rightarrow E[13]$. Un tel point donne lieu à $\left(13^{2}-1\right) / 2=84$ points de $Y_{1}(13)\left(\mathbb{Q}\left(\mu_{13}\right)\right)$. Notons $\mathcal{P}_{(E, \pi)}$ cet ensemble de points. Supposons que pour tout $x$ de $\mathcal{P}_{(E, \pi)}, W_{13} . x$ n'est pas dans $\mathcal{P}_{(E, \pi)}$. Alors le sous-ensemble $\mathcal{P}_{(E, \pi)} \cup W_{13} \cdot \mathcal{P}_{(E, \pi)}$ de $Y_{1}(13)\left(\mathbb{Q}\left(\mu_{13}\right)\right)$ est de cardinal $2 \times 84=168>12^{2}$, ce qui est impossible d'après la proposition 2 .

Par conséquent, il existe $x \in \mathcal{P}_{(E, \pi)}$ tel que $y=W_{13} . x \in \mathcal{P}_{(E, \pi)}$. Autrement dit, il existe $P, Q$ deux points d'ordre 13 de $E$ définis sur $\mathbb{Q}\left(\mu_{13}\right)$ et un isomorphisme défini sur $\mathbb{Q}\left(\mu_{13}\right)$ envoyant $(E, Q)$ sur

$$
W_{13} .(E, P)=\left(E /\langle P\rangle, P^{\prime}+\langle P\rangle\right),
$$

où $P^{\prime}$ est le point d'ordre 13 de $E$ tel que $e_{13}\left(P^{\prime}, P\right)=e^{2 i \pi / 13}$, l'application $e_{13}: E[13] \times E[13] \rightarrow \mu_{13}$ étant l'accouplement de Weil.

En particulier, on dispose d'une isogénie $\psi$ de $E$ dans $E$ de degré 13 définie sur $\mathbb{Q}\left(\mu_{13}\right)$. La courbe elliptique $E$ est donc à multiplication complexe. L'ensemble des endomorphismes End $E$ de $E$ sur $\mathbb{C}$ est isomorphe 
à un ordre $R$ d'un corps quadratique imaginaire. Soit [.] $R \rightarrow$ End $E$ l'isomorphisme normalisé, et $\alpha \in R$ tel que $\psi=[\alpha]$. L'isogénie $[\alpha]$ étant définie sur $\mathbb{Q}\left(\mu_{13}\right)$ ainsi que la courbe elliptique $E$, on a $\alpha \in \mathbb{Q}\left(\mu_{13}\right)$. Or $13 \equiv 1(\bmod 4)$, donc $\mathbb{Q}\left(\mu_{13}\right)$ ne contient aucun corps quadratique imaginaire, et donc $\alpha \in \mathbb{Q}$. C'est impossible car sinon, $13=\operatorname{deg}[\alpha]=\left|N_{\mathbb{Q}}^{K}(\alpha)\right|$ serait un carré. Ceci achève la preuve.

4. Remarque. Lorsque j'ai exposé cette démonstration pendant les vingt-deuxièmes Journées Arithmétiques (juin 2001), B. Poonen m'a signalé un résultat de R. F. Coleman qui, en utilisant la finitude du groupe $J_{1}(13)\left(\mathbb{Q}\left(\mu_{13}\right)\right)$, fournit une borne plus petite du cardinal de $Y_{1}(13)\left(\mathbb{Q}\left(\mu_{13}\right)\right)$, et simplifie alors la démonstration du théorème.

Rappelons le résultat de Coleman :

ThÉORÈme 2 (Coleman, [1]). Soient $C$ une courbe de genre $g$ définie sur un corps de nombres $K$, et $J$ sa jacobienne. Supposons que le rang de $J(K)$ soit au plus $g-1$. Soit $\mathcal{P}$ un idéal non ramifié de $K$ en lequel $C$ a bonne réduction et de caractéristique résiduelle supérieure à $2 g$. Notons $f_{\mathcal{P}}$ le degré résiduel en $\mathcal{P}$. Alors

$$
|C(K)| \leq f_{\mathcal{P}}+2 g\left(\sqrt{f_{\mathcal{P}}}+1\right)-1 .
$$

Appliquons ce théorème à $C=X_{1}(13), K=\mathbb{Q}\left(\mu_{13}\right), \mathcal{P} \mid 53$, pour lesquels les hypothèses sont vérifiées, en particulier grâce au lemme 1 qui assure que le rang de $J_{1}(13)\left(\mathbb{Q}\left(\mu_{13}\right)\right)$ est inférieur à $g-1=1$. On obtient $\left|X_{1}(13)\left(\mathbb{Q}\left(\mu_{13}\right)\right)\right| \leq 85$, donc $\left|Y_{1}(13)\left(\mathbb{Q}\left(\mu_{13}\right)\right)\right| \leq 73$. On raisonne alors par l'absurde comme dans la section 3 : un point de $Y(13)\left(\mathbb{Q}\left(\mu_{13}\right)\right)$ donnerait lieu à 84 points de $Y_{1}(13)\left(\mathbb{Q}\left(\mu_{13}\right)\right)$, ce qui est impossible d'après ce qui précède.

\section{Références}

[1] R. F. Coleman, Effective Chabauty, Duke Math. J. 52 (1985), 765-770.

[2] J. E. Cremona, Modular symbols for $\Gamma_{1}(N)$ and elliptic curves with everywhere good reduction, Math. Proc. Cambridge Philos. Soc. 111 (1992), 199-218.

[3] F. Diamond and J. Im, Modular forms and modular curves, dans : Seminar on Fermat's Last Theorem, vol. 17, Toronto, ON, 1993-1994, 39-133.

[4] K. Kato, p-adic Hodge theory and values of zeta functions of modular forms, à paraître.

[5] J.-C. Lario and J. Quer, Table of some Hecke operator's eigenvalues, non publiée.

[6] Yu. Manin, Parabolic points and zeta function of modular curves, Math. USSR-Izv. 6 (1972), 19-64.

[7] L. Merel, Sur la nature non cyclotomique des points d'ordre fini des courbes elliptiques, Duke Math. J. 110 (2001), 81-119.

[8] - Universal Fourier expansions of modular forms, dans : On Artin's Conjecture for Odd 2-Dimensional Representations, Lecture Notes in Math. 1585, Springer, 1994, 59-94. 
[9] L. Merel and W. Stein, The field generated by the points of small prime order on an elliptic curve, Internat. Math. Res. Notices 2001, no. 20, 1075-1082.

[10] A. P. Ogg, Rational points on certain elliptic modular curves, mimeographed notes, Berkeley, St. Louis, 1972.

[11] P. Parent, Torsion des courbes elliptiques sur les corps cubiques, Ann. Inst. Fourier (Grenoble) 50 (2000), 723-749.

[12] D. E. Rohrlich, Modular curves, Hecke correspondences, and L-functions, dans : Modular Forms and Fermat's Last Theorem, G. Cornell, J. H. Silverman and G. Stevens (eds.), Springer, 1997, 41-100.

[13] J. Tilouine, Hecke algebras and the Gorenstein property, ibid., 327-342.

Université Paris VI

175 rue du Chevaleret

75013 Paris, France

E-mail: rebolledo@math.jussieu.fr 